\title{
Centella asiatica protects against UVB-induced HaCaT keratinocyte damage through microRNA expression changes
}

\author{
IN-SOOK AN ${ }^{1 *}$, SUNGKWAN AN $^{1,2^{*}}$, TAE-BOO CHOE ${ }^{3}$, SANG-MO KANG $^{3}$, JAE HO LEE $^{4}$, \\ IN-CHUL PARK ${ }^{5}$, YOUNG-WOO JIN ${ }^{6}$, SU-JAE LEE ${ }^{7}$ and SEUNGHEE BAE ${ }^{2}$ \\ ${ }^{1}$ Korea Institute for Skin and Clinical Sciences, ${ }^{2}$ Molecular-Targeted Drug Research Center and \\ ${ }^{3}$ Department of Microbial Engineering, Konkuk University, Seoul 143-701; ${ }^{4}$ Laboratory of Molecular Oncology, \\ Cheil General Hospital and Women's Healthcare Center, Kwandong University, College of Medicine, Seoul 100-380; \\ ${ }^{5}$ Laboratory of Functional Genomics, Korea Institute of Radiological and Medical Sciences, Seoul 139-706; \\ ${ }^{6}$ Division of Radiation Effect Research, Radiation Health Research Institute of KHNP, Seoul 132-703; \\ ${ }^{7}$ Department of Chemistry, Hanyang University, Seoul 133-791, Republic of Korea
}

Received June 7, 2012; Accepted September 10, 2012

DOI: $10.3892 / \mathrm{ijmm} .2012 .1157$

\begin{abstract}
This study aimed to evaluate the protective effects of Centella asiatica (C. asiatica) against ultraviolet B (UVB) damage in human keratinocytes using microRNA (miRNA) expression profiling analysis. Titrated extract of $C$. asiatica (TECA) demonstrated low cytotoxicity in normal human HaCaT keratinocytes only at low doses $(<5 \mu \mathrm{g} / \mathrm{ml})$. UVB $\left(50 \mathrm{~mJ} / \mathrm{cm}^{2}\right)$ irradiation significantly decreased cell viability, and TECA treatment decreased the UVB toxicity. By using miRNA microarrays, we determined that 72 miRNAs had an altered expression following TECA treatment in UVB-irradiated keratinocytes (46 upregulated and 26 downregulated). Using an miRNA target gene prediction tool and Gene Ontology (GO) analysis, we determined that miRNAs with altered expression were functionally related with the inhibition of apoptosis and cell proliferation. Overall, these results provide meaningful information to facilitate the understanding of TECA-mediated UVB protection in human keratinocytes.
\end{abstract}

\section{Introduction}

UV irradiation leads to cell aging, senescence, apoptosis and cancer in human keratinocytes by inducing reactive oxygen species (ROS), DNA damage and inflammatory and immunological reactions (1). Members of the mitogen-

Correspondence to: Dr Seunghee Bae, Molecular-Targeted Drug Research Center, Konkuk University, 1 Hwayang-dong, Gwangjin-gu, Seoul 143-701, Republic of Korea

E-mail: sbae@konkuk.ac.kr

${ }^{*}$ Contributed equally

Key words: Centella asiatica, keratinocyte, ultraviolet B, miRNA expression, cell viability activated protein kinase (MAPK) family, including ERK1/2, p38 MAPK and JNK, are phosphorylated (activated) by UV irradiation (1). A low-dose of UVB (ultraviolet B) $\left(0.1 \mathrm{~kJ} / \mathrm{cm}^{2}\right)$ significantly produces tumor necrosis factor $\alpha(\mathrm{TNF}-\alpha)$, which is involved in the suppression of contact hypersensitivity and in the decreased immunosurveillance in UV-damaged keratinocytes (2). In addition, the protein half-life of nuclear factor $-\kappa \mathrm{B}(\mathrm{NF}-\kappa \mathrm{B})$ is prolonged by the inhibitor of $\mathrm{NF}-\kappa \mathrm{B} \alpha$ $(\mathrm{I} \kappa \mathrm{B} \alpha)$ phosphorylation and proteasomal degradation, and activated NF- $\kappa \mathrm{B}$ may induce both anti- and pro-apoptotic pathways (3-7). Furthermore, UVB irradiation induces cytokine IL-21 and nitric oxide (NO), which are closely related to keratinocyte immune responses $(8,9)$. Collectively, UVB irradiation activates multiple signaling cascades in keratinocytes.

microRNAs (miRNAs) are small, non-coding RNA molecules that directly regulate the expression of target mRNA transcripts (10). miRNAs have been reported to be involved in almost all cellular processes, including development, proliferation, immune response, metabolism and cell death (11-13). In human skin, miRNA-based studies first investigated miRNA expression patterns in normal human skin and melanocytic nevi (14,15). A functional relationship between keratinocyte miRNAs and psoriasis was reported, and it was determined that miR-125b modulates abnormal keratinocyte proliferation in psoriasis by targeting FGFR2 $(16,17)$. The miRNA expression pattern during human keratinocyte differentiation has also been investigated (18). One recent study analyzed UVB-dependent miRNA expression profile changes in keratinocytes (19). Overall, these reports indicate that miRNAs may be key regulators of multiple cellular processes in keratinocytes and further suggest that miRNAs may have protective functions in response to UVB.

Centella asiatica (C. asiatica, also known as gotu kola) is a plant used in traditional herbal medicine with pharmacological effects on skin wound healing $(20,21)$. It also shows anti-oxidant, anti-microbial and anticancer properties $(22,23)$. We demonstrated that $\mathrm{H}_{2} \mathrm{O}_{2}$-induced cell senescence was inhibited by treating human dermal fibroblasts with a 
titrated extract of $C$. asiatica (TECA) (24). Although there was a recent report regarding the UVB protective effect of TECA treatment on dermal fibroblasts (25), the molecular mechanisms of UV protection have not been elucidated. Furthermore, a possible UV protective effect on human keratinocytes has not been studied. In this current study, we demonstrate that TECA exerts novel UVB protection in human keratinocytes and characterized miRNA expression profiles that correspond to TECA-mediated UVB protection.

\section{Materials and methods}

Cell culture. Normal human HaCaT keratinocytes were maintained in Dulbecco's modified Eagle's medium (DMEM; Gibco-Invitrogen Life Technologies, Carlsbad, CA, USA) supplemented with $10 \%$ fetal bovine serum (FBS; SigmaAldrich, St. Louis, MO, USA) with penicillin/streptomycin.

UVB irradiation and TECA treatment. A day before UVB irradiation, $\mathrm{HaCaT}$ cells $\left(4 \times 10^{3}\right)$ were seeded into 96-well plates. For RNA purification, $7 \times 10^{5}$ cells were seeded into 60-mm dishes. Before UVB irradiation, cells were pre-treated with the control dimethyl sulfoxide (DMSO; Sigma-Aldrich) or TECA (Bayer Health Care, Berlin, Germany) for $3 \mathrm{~h}$. Cells were washed with phosphate-buffered saline (PBS) and exposed to $50 \mathrm{~mJ} / \mathrm{cm}^{2}$ UVB without dish covers. After irradiation, the cells were cultured in DMEM media containing $10 \%$ FBS with DMSO or TECA for $24 \mathrm{~h}$.

RNA purification and qualification. Total RNA was extracted and purified with TRIzol reagent (Invitrogen Life Technologies) according to the manufacturer's instructions. The integrity of each RNA sample was verified with an Agilent 2100 Bioanalyzer (Agilent Technologies, Santa Clara, CA, USA). The quality and concentration of each RNA sample were determined using MaestroNano (Maestrogen, Las Vegas, NV, USA). RNA quality parameters for miRNA microarray analysis were A260/280 and A260/A230 values >1.8 and an RNA integrity no. (RIN) $>8.0$.

Microarray analysis of miRNA profiles. The miRNA profiling analysis was performed using SurePrint G3 Human V16 miRNA 8 x 60K (Agilent Technologies) containing a probe for 1,205 and 144 human viral miRNAs. Each qualified RNA sample (100 ng) was dephosphorylated with calf intestinal alkaline phosphatase (CIP) at $37^{\circ} \mathrm{C}$ for $30 \mathrm{~min}$. Then, the dephosphorylated RNA samples were labeled with cyanine 3 -pCp using T4 RNA ligase by incubating at $16^{\circ} \mathrm{C}$ for $2 \mathrm{~h}$. After the labeling reaction, the samples were completely dried using a vacuum concentrator at $55^{\circ} \mathrm{C}$ for $4 \mathrm{~h}$. The dried samples were treated with GE Blocking Agent (Agilent Technologies) and hybridized to the probes on the microarray at $55^{\circ} \mathrm{C}$, at $20 \mathrm{rpm}$ in the Agilent Microarray Hybridization Chamber (Agilent Technologies) for $20 \mathrm{~h}$. The microarray slide was washed and scanned with the Agilent scanner to obtain the microarray image. The numerical data for the miRNA profiles were extracted from the image with the Feature Extraction program (Agilent Technologies). These data were analyzed with GeneSpring GX software version 7.3 (Agilent Technologies). miRNAs whose flags were present in at least one sample were filtered and applied to the fold-change analysis, which was conducted by a factor of 1.5 -fold between two groups: UVB-exposed/DMSO-treated control and UVB-exposed $/ 2 \mu \mathrm{g} / \mathrm{ml}$ TECA-treated HaCaT keratinocytes.

Bioinformatical analysis of miRNAs. Meaningful altered miRNAs were selected, and their putative cellular target genes were determined using MicroCosm Target version 5 (www. ebi.ac.uk/enright-srv/microcosm/thdoc/targets/v5/). The target genes were categorized into four groups (aging, apoptosis, cell proliferation and skin development) using the Gene Ontology (GO) analysis tool AmiGO (amigo.geneontology.org/cgi-bin/ amigo/browse.cgi). Further GO analysis was performed for several categories, such as anti-apoptosis, MAPKK activity, Ras protein signal transduction, small GTPase-mediated signal transduction, positive or negative regulation of cell growth, cell proliferation, cell cycle, immune response and positive regulation of p53-mediated signaling.

\section{Results}

TECA protects HaCaT cells against UVB damage. We first assessed the cytotoxicity of TECA. HaCaT cells were treated with increasing doses of TECA $(1,2,5,10$ or $20 \mu \mathrm{g} / \mathrm{ml})$ for $24 \mathrm{~h}$, and the WST-1-based cellular toxicity assay was used to determine cell viability. As shown in Fig. 1A, low doses (up to $5 \mu \mathrm{g} / \mathrm{ml}$ ) of TECA had no significant cytotoxic effect on $\mathrm{HaCaT}$ cells, while higher doses $(10$ and $20 \mu \mathrm{g} / \mathrm{ml})$ were more cytotoxic. Based on these results, we used 1, 2 and $5 \mu \mathrm{g} / \mathrm{ml}$ treatments in further experiments. Next, the protective activity of TECA on keratinocytes was determined. HaCaT cells pre-treated with TECA were exposed to $50 \mathrm{~mJ} / \mathrm{cm}^{2}$ of UVB without any protective covers. After UVB-irradiation, the cells were incubated with the indicated doses of TECA for $24 \mathrm{~h}$. The WST-1 assay showed that the irradiated cells without TECA displayed a $43.52 \%$ cell survival rate, compared to non-irradiated control cells and TECA treatment $(2 \mu \mathrm{g} / \mathrm{ml})$ markedly improved the cell survival rate to $71.25 \%$ (Fig. 1B). Overall, TECA prevented UVB-mediated keratinocyte cell death.

TECA alters miRNA expression profiles in UVB-treated keratinocytes. We further determined the protective effect of TECA with a miRNA expression profiling analysis and observed different miRNA expression patterns in response to TECA treatment in UVB-irradiated HaCaT cells. As shown in Fig. 2A, 72 human miRNAs were altered $>1.5$-fold in the TECA-treated, UVB-irradiated HaCaT cells, compared to those that were only exposed to UVB. The full list of 72 miRNAs is shown in Table I. TECA treatment affected miRNA expression levels, but the fold change was not $>4.0$. Among the altered miRNAs, 46 miRNAs were upregulated after TECA treatment and 26 miRNAs were downregulated (Fig. 2B). However, the extent of changes varied among miRNAs; the expression levels of miR-636, miR-3620 and miR-296-5p were significantly increased by 3.51-, 3.60- and 2.54-fold, respectively, whereas the expression of miR-622 and miR-455-5p was significantly decreased by $2.82-$ and 2.07-fold, respectively. Overall, TECA treatment influenced certain miRNA expression levels, suggesting that specific 
A

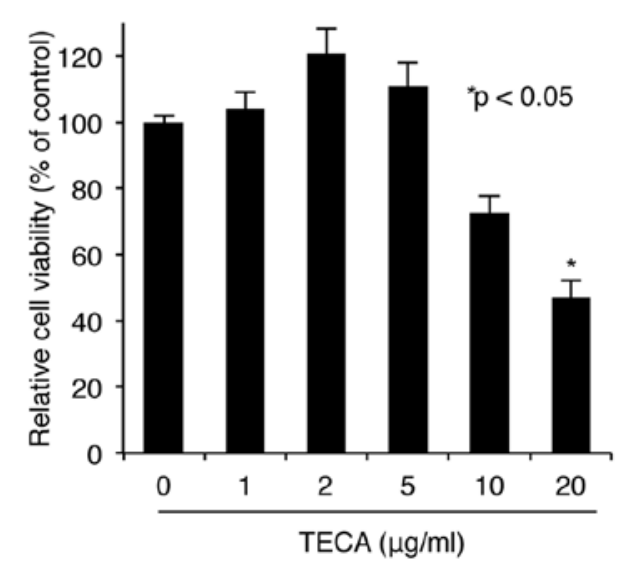

B

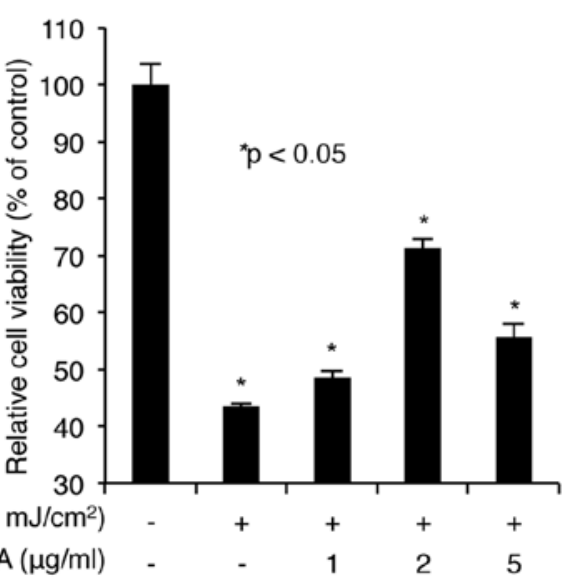

Figure 1. Cytotoxic and UVB protective activities of TECA in HaCaT keratinocytes. (A) Cytotoxicity assay. HaCaT cells (4x103) were seeded on 96-well plates, and TECA was applied for $24 \mathrm{~h}$ as indicated. The cellular toxicity was measured using a WST-1 assay. The results are representative of 3 independent experiments (means \pm SD are shown). (B) UVB protective activity of TECA in HaCaT cells. The graph represents the means \pm SD of relative cell viability in each sample from triplicate experiments. For both assays, Student's t-tests were performed to assess statistical significance (" $\mathrm{p}<0.05$ compared with the control).

A

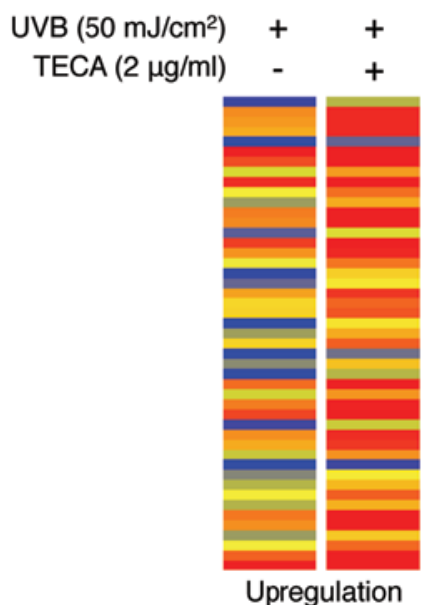

B

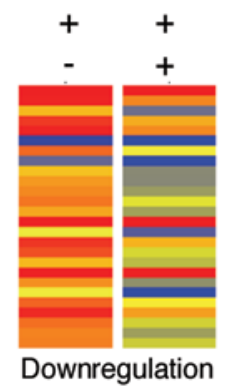

Expression level

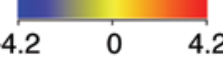

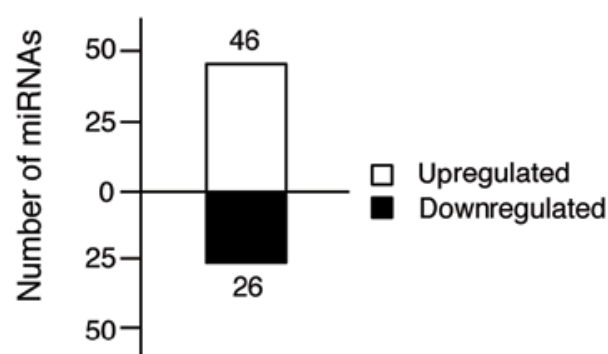

Figure 2. Analysis of TECA-induced miRNA expression profiles in UVB-irradiated HaCaT cells. (A) TECA induced both the upregulation and downregulation of miRNA expression levels. miRNAs with $>1.5$-fold expression changes were selected for further analysis. Red and blue bars indicate increased and decreased expression, respectively. (B) The number of miRNAs altered by $>1.5$-fold by TECA treatment in UVB-irradiated HaCaT cells.

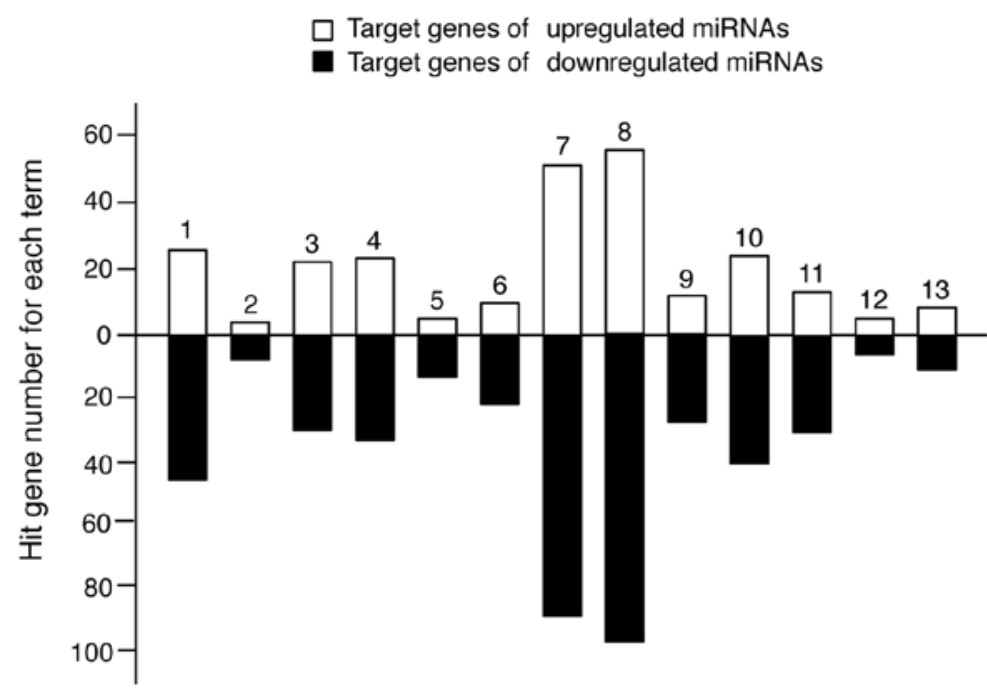

1. Anti-apoptosis

2. Activation of MAPKK activity

3. Ras protein signal transduction

4. Small GTPase mediated signal transduction

5. Positive regulation of cell growth

6. Negative regulation of cell growth

7. Positive regulation of cell proliferation

8. Negative regulation of cell proliferation

9. Positive regulation of cell cycle

10. Negative regulation of cell cycle

11. Positive regulation of immune response

12. Negative regulation of immune response

13. Positive regulation of p53-mediated signaling

Figure 3. Gene Ontology (GO)-based categorization of miRNA-target genes. The y-axis values display the number of genes involved in each of the processes listed on the right. 
Table I. miRNAs altered by TECA treatment in UVB-exposed HaCaT keratinocytes.

\begin{tabular}{|c|c|c|c|c|c|}
\hline miR name & $\mathrm{FC}$ & Chromosome & miR name & $\mathrm{FC}$ & Chromosome \\
\hline ebv-miR-BHRF1-1 & 1.57 & - & hsa-miR-636 & 3.51 & Chr17 \\
\hline hsa-let-7b* & 1.53 & Chr22 & hsa-miR-664 & 1.54 & Chr1 \\
\hline hsa-let-7f-1 & 1.59 & Chr9 & hsa-miR-767-3p & 1.56 & ChrX \\
\hline hsa-miR-1225-3p & 1.70 & Chr16 & hsa-miR-92b & 1.71 & Chr1 \\
\hline hsa-miR-1227 & 1.57 & Chr19 & hsa-miR-933 & 1.63 & Chr2 \\
\hline hsa-miR-1228 & 1.55 & Chr12 & hsv1-miR-H6-3p & 1.61 & - \\
\hline hsa-miR-1234 & 1.66 & Chr8 & hsv1-miR-H7* & 1.65 & - \\
\hline hsa-miR-1237 & 1.54 & Chr11 & hsv1-miR-H20 & 1.61 & - \\
\hline hsa-miR-1238 & 1.58 & Chr19 & hsv1-miR-H7-3p & 1.66 & - \\
\hline hsa-miR-129* & 1.61 & Chr7 & kshv-miR-K12-8* & 1.63 & - \\
\hline hsa-miR-1470 & 1.80 & Chr8 & hsa-miR-192 & -1.78 & Chr11 \\
\hline hsa-miR-1539 & 1.62 & Chr18 & hsa-miR-182 & -1.84 & Chr7 \\
\hline hsa-miR-1825 & 1.66 & Chr20 & hsa-miR-210 & -2.06 & Chr11 \\
\hline hsa-miR-18b* & 1.55 & ChrX & hsa-miR-132 & -2.00 & Chr17 \\
\hline hsa-miR-191* & 1.53 & Chr3 & hsa-miR-9* & -2.08 & Chr1 \\
\hline hsa-miR-2116* & 1.59 & Chr15 & hsa-miR-125a-5p & -1.53 & Chr19 \\
\hline hsa-miR-223 & 1.64 & ChrX & hsa-miR-155 & -1.58 & Chr21 \\
\hline hsa-miR-296-5p & 2.54 & Chr20 & hsa-miR-99b & -1.80 & Chr19 \\
\hline hsa-miR-3180-5p & 1.72 & Chr16 & hsa-miR-362-5p & -1.72 & ChrX \\
\hline hsa-miR-33b ${ }^{*}$ & 1.54 & Chr17 & hsa-miR-374a & -1.54 & ChrX \\
\hline hsa-miR-3613-3p & 1.52 & Chr13 & hsa-miR-494 & -1.53 & Chr14 \\
\hline hsa-miR-3614-5p & 1.61 & Chr17 & hsa-miR-181d & -1.70 & Chr19 \\
\hline hsa-miR-3620 & 3.60 & Chr1 & hsa-miR-532-5p & -2.07 & ChrX \\
\hline hsa-miR-3675-3p & 1.78 & Chr1 & hsa-miR-455-5p & -2.07 & Chr9 \\
\hline hsa-miR-3676 & 1.53 & Chr17 & hsa-miR-622 & -2.82 & Chr13 \\
\hline hsa-miR-3679-3p & 1.54 & Chr2 & hsa-miR-625 & -1.65 & Chr14 \\
\hline hsa-miR-3940 & 1.77 & Chr19 & hsa-miR-660 & -1.63 & ChrX \\
\hline hsa-miR-423-3p & 2.04 & Chr17 & ebv-miR-7-1* & -1.53 & - \\
\hline hsa-miR-425* & 1.59 & Chr3 & hsa-miR-7-1 ${ }^{*}$ & -1.88 & Chr9 \\
\hline hsa-miR-4274 & 1.61 & Chr4 & hsa-miR-181a-2* & -1.51 & Chr9 \\
\hline hsa-miR-4310 & 1.52 & Chr15 & hsa-miR-140-3p & -1.56 & Chr16 \\
\hline hsa-miR-4313 & 1.58 & Chr15 & hsa-miR-362-3p & -2.06 & ChrX \\
\hline hsa-miR-4323 & 1.62 & Chr19 & hsa-miR-423-5p & -1.65 & Chr17 \\
\hline hsa-miR-550a & 1.51 & Chr17 & hsa-miR-483-5p & -1.54 & Chr11 \\
\hline hsa-miR-602 & 1.57 & Chr9 & hsa-miR-3652 & -1.78 & Chr12 \\
\hline hsa-miR-634 & 1.69 & Chr17 & & & \\
\hline
\end{tabular}

The list shows miRNAs exhibiting a $>1.5$-fold expression change after flag sorting. FC, fold change.

cellular response mechanisms may be involved in TECAmediated UVB protection of keratinocytes.

Bioinformatical analysis of TECA-specific miRNAs and their putative targets. We next assessed the biological meaning of the altered miRNA expression in UVB protection. miRNAs post-transcriptionally regulate gene expression by binding to target mRNAs, indicating that the biological functions of miRNAs are dependent on that of their target genes (26).

First, we analyzed the putative target genes of miRNAs that were meaningfully altered by TECA treatment using the miRNA target prediction bioinformatical tool MicroCosm. We observed that 1,354 and 1,975 genes were putatively targeted by the upregulated and downregulated miRNAs, respectively $(\mathrm{p}<0.05$, data not shown). We next analyzed the biological functions for each target gene with the GO analytical tool AmiGO. Since UV irradiation induces cell aging and apoptosis (1), we sorted the target genes into several categories, including aging, apoptosis, cell proliferation and skin development (Tables II and III). We revealed that a number of target genes were involved in these four processes, suggesting that the effects of TECA may be functionally related to UV protective properties by affecting the protein products of those genes. For example, miR-636, which was increased by 3.51-fold by TECA, putatively targets genes such as suppressor of cytokine signaling 3 (SOCS3), 
Table II. Predicted target genes of the miRNAs upregulated in response to TECA treatment in UVB-exposed HaCaT keratinocytes.

\begin{tabular}{|c|c|c|c|c|}
\hline \multirow[b]{2}{*}{ miRNA } & \multicolumn{4}{|c|}{ Target gene functions } \\
\hline & Aging & Apoptosis & Cell proliferation & $\begin{array}{c}\text { Skin } \\
\text { development }\end{array}$ \\
\hline hsa-miR-1228 & TP53 & TP53, CEBPG, PLAGL2, TJP1 & $\begin{array}{l}\text { TP53, ATP8A2, CD47, } \\
\text { MKI67, SSTR1 }\end{array}$ & - \\
\hline hsa-miR-1237 & ID2 & $\begin{array}{l}\text { BCL6, ERBB3, PAK7, } \\
\text { ANKRD13C, SGPL1, UBE2Z }\end{array}$ & $\begin{array}{l}\text { ID2, BCL6, ERBB3, PAK7, } \\
\text { NFIB, CCND2 }\end{array}$ & - \\
\hline hsa-miR-1825 & $\begin{array}{l}\text { SERPINE1, } \\
\text { ULK3 }\end{array}$ & $\begin{array}{l}\text { SERPINE1, ITCH, OSR1, CECR2, } \\
\text { PREX1, ROCK1, TIAM1, } \\
\text { BCL11B, GPI, MITF, PKN2 }\end{array}$ & $\begin{array}{l}\text { SERPINE1, ITCH, OSR1, } \\
\text { CHRNB2, EPS15, HHIP, } \\
\text { KIT, MAB21L1, R3H1, } \\
\text { FOXO4, BCL11B, MITF }\end{array}$ & $\begin{array}{l}\text { COL5A3, } \\
\text { BCL11B }\end{array}$ \\
\hline hsa-miR-223 & F3, RPS6KB1 & $\begin{array}{l}\text { F3, RPS6KB1, APC, FGFR2, } \\
\text { FOXO1, FOXO3, IGF1R, ECT2, } \\
\text { ATP7A, HSP90B1, NLRP3, RASA1, } \\
\text { RHOB, RNF34, SNCA, SYNGAP1 }\end{array}$ & $\begin{array}{l}\text { F3, RPS6KBI, APC, FGFR2, } \\
\text { FOXO1, FOXO3, IGF1R, } \\
\text { ACVR2A, CBLB, MYH10, } \\
\text { PDS5B, SCARB1, WDR77, NFIB }\end{array}$ & $\begin{array}{l}\text { APC, } \\
\text { ATP7A }\end{array}$ \\
\hline hsa-miR-296-5p & $\begin{array}{l}\text { BBC } 3, \text { HMGA1, } \\
\text { PRELP }\end{array}$ & $\begin{array}{l}\text { BBC3, CNTFR, FGFR1, } \\
\text { NUAK2, MEF2D }\end{array}$ & $\begin{array}{l}\text { HMGA1, CNTFR, FGFR1, } \\
\text { CXCL10, MLL2 }\end{array}$ & - \\
\hline hsa-miR-550a & CDK6, TERF2 & $\begin{array}{l}\text { ARHGEF12, MTDH, PSME1, } \\
\text { UNC5A, SOX4 }\end{array}$ & $\begin{array}{l}\text { CDK6, APPL2, PDGFRA, } \\
\text { TRIM27, SOX4, SOX11 }\end{array}$ & - \\
\hline hsa-miR-634 & P2RY2, TGFBR1 & $\begin{array}{l}\text { ADRB2, ERBB4, VAV3, CSDA, IAPP, } \\
\text { NEUROD1, RASSF5, YWHAE, } \\
\text { KCNMA1, TGFBR1, KPNA1, } \\
\text { MAP3K1, BLOC1S2, PDCD6IP }\end{array}$ & $\begin{array}{l}\text { ADRB2, ERBB4, VAV3, CKS1B, } \\
\text { FOXP2, GOLPH3, INSR, JAG1, } \\
\text { CCND2, TGFBR1, FBXW7, } \\
\text { TBC1D8, EVI1, SSR1 }\end{array}$ & - \\
\hline hsa-miR-636 & SOCS3 & $\begin{array}{l}\text { TCF7L2, RPS6KA2, SFRP2, TGFBR2, } \\
\text { TRAF5, ACTN1, ARF6, GRIK2, } \\
\text { ITSN1, PCGF2, PROC, RPS6KA3, } \\
\text { RTN3, CBL, SENP1, YWHAZ, } \\
\text { SOCS3, MITF, PKN2, PRKCE }\end{array}$ & $\begin{array}{l}\text { TCF7L2, RPS6KA2, SFRP2, } \\
\text { TGFBR2, TRAF5, BCAT1, EMX2, } \\
\text { LIFR, RNF139, MITF, TOB1 }\end{array}$ & TCF7L2 \\
\hline hsa-miR-664 & - & $\begin{array}{l}\text { CUL3, ZMYND11, BMX, C1D, } \\
\text { CYCS, NET1, PDPK1, TAF9, } \\
\text { TNFAIP1, UBE2D3, PAX3 }\end{array}$ & $\begin{array}{l}\text { CUL3, ZMYND11, ACSL6, BAP1, } \\
\text { CDC14A, MTCP1, SMAD4, IRF2, } \\
\text { PHOX2B, PAX3, FOXO4 }\end{array}$ & - \\
\hline hsa-miR-767-3p & AGT, HTR2A & $\begin{array}{l}\text { AGT, CSNK2A1, CSNK2A2, } \\
\text { HSPA9, SET, TRAF7, UBE2B, } \\
\text { BLOC1S2, PDCD6IP }\end{array}$ & $\begin{array}{l}\text { AGT, HTR2A, ASPH, CDC25C, } \\
\text { FGF7, JARID2, MLXIPL, NPR1, } \\
\text { PDPN, PTCH1, ST8SIA1, TENC1, } \\
\text { TIMP2, UBR5, BLOC1S2, } \\
\text { CNOT8, EVI1, SSR1 }\end{array}$ & - \\
\hline hsa-miR-92b & $\begin{array}{l}\text { ADRB1, MORC3, } \\
\text { NOX } 4, \text { HCN2, } \\
\text { PTEN }\end{array}$ & $\begin{array}{l}\text { ADRB1, APPL1, BTG2, GPI, } \\
\text { CDK5R1, HAND2, ITGAV, KLF4, } \\
\text { PTPRJ, SGK3, USP28, ARHGEF17, } \\
\text { BCL2L11, DYRK2, FXR1, HIPK3, } \\
\text { ITGA6, LYST, MAP2K4, NR4A3, } \\
\text { RAD21, ROBO2, TRAF3, PTEN, } \\
\text { SOX4, DAB2IP, BCL11B, PAX3 }\end{array}$ & $\begin{array}{l}\text { MORC3, NOX4, APPL1, BTG2, } \\
\text { CDK5R1, HAND2, ITGAV, KLF4, } \\
\text { BMPR2, EVI5, CDC27, CDCA7L, } \\
\text { TSC1, CHRM5, CDKN1C, SGK3, } \\
\text { FOSL2, PTPRJ, CXCL5, GATA2, } \\
\text { GDF11, MS4A2, NKX2-3, PTPRU, } \\
\text { S1PR1, TACC2, TGIF1, TOB2, } \\
\text { DAB2IP, ZEB2, PTEN, ODZ1, } \\
\text { BCL11B, SOX4, SOX11, PAX3 }\end{array}$ & $\begin{array}{l}\text { COL1A2, } \\
\text { BCL11B }\end{array}$ \\
\hline
\end{tabular}

microphthalmia-associated transcription factor (MITF), empty spiracles homeobox 2 (EMX2) and transcription factor 7-like 2 (TCF7L2). Conversely, miR-622 was decreased by 2.82-fold by TECA and putatively targets genes included nucleophosmin (NPM1), E2F transcription factor 1 (E2F1) and peroxisome proliferator-activated receptor $\delta$ (PPARD). 
Table III. Predicted target genes of the miRNAs downregulated in response to TECA treatment in UVB-exposed HaCaT keratinocytes.

\begin{tabular}{|c|c|c|c|c|}
\hline \multirow[b]{2}{*}{ miRNA } & \multicolumn{4}{|c|}{ Target gene functions } \\
\hline & Aging & Apoptosis & Cell proliferation & $\begin{array}{c}\text { Skin } \\
\text { development }\end{array}$ \\
\hline hsa-miR-192 & SMC5 & DICER1, GDNF & DICER1, EREG & - \\
\hline hsa-miR-182 & TMEM115, RTN4 & $\begin{array}{l}\text { RTN4, CITED2, EGR3, MEF2C, } \\
\text { BCL2L13, ELMO1, NF1, MLL, } \\
\text { ARHGEF3, BCL2L12, CREB1, } \\
\text { MITF, SOX2, AATK, ACVR1, } \\
\text { RAC1, TNFAIP8, TOX3 }\end{array}$ & $\begin{array}{l}\text { TMEM115, CITED2, NF1, } \\
\text { EGR3, MEF2C, TNFSF11, } \\
\text { MITF, SOX2, CBFA2T3, } \\
\text { EVI1, MTSS1, PYGO2, } \\
\text { CDV3, TOB1, FGF9 }\end{array}$ & - \\
\hline hsa-miR-132 & CTGF & $\begin{array}{l}\text { FOXO3, MAPT, APAF1, RB1, } \\
\text { SYNGAP1, MAPK3, CTGF }\end{array}$ & $\begin{array}{l}\text { ZEB2, FOXO3, HBEGF, } \\
\text { CTGF, RB1, SPRY1 }\end{array}$ & - \\
\hline hsa-miR-125a-5p & $\begin{array}{l}\text { BAK1, VDR, } \\
\text { CASP2, MAPK14 }\end{array}$ & $\begin{array}{l}\text { SGPL1, SYVN1, TAF9N, TIAF1, } \\
\text { BAK1, VDR, CASP2, MAPK2, } \\
\text { IER3IP1, KCNIP3, MYO18A, } \\
\text { TRIAP1, MCL1, MAP3K11, } \\
\text { SSTR3, ECE1, HK2, APC, } \\
\text { BCL11B, COL4A3, IL6R, }\end{array}$ & $\begin{array}{l}\text { BCL11B, COL4A3, GPC4, } \\
\text { BCAT1, CDH5, CGREF1, } \\
\text { CYP27B1, DIS3L2, LIPA, BAK1, } \\
\text { VDR, APC,IL6R, PRDM1, } \\
\text { ENPEP, ESRRA, FBW4, } \\
\text { TNFAIP3, MAP3K11, TNFSF4S }\end{array}$ & $\begin{array}{l}\text { COL4A3, } \\
\text { BCL11B, } \\
\text { APC }\end{array}$ \\
\hline hsa-miR-155 & FOS & $\begin{array}{l}\text { MAP3K10, RPS6KA3, VAV3, } \\
\text { NKX3-1, TRIM32, MEF2A, } \\
\text { PHF17, PKN2, SGK3, CBL, } \\
\text { TP53INP1, YWHAZ }\end{array}$ & $\begin{array}{l}\text { NKX3-1, TRIM32, VAV3, SMAD2, } \\
\text { SMAD1, SGK3, CSF1R, } \\
\text { FGF7, JARID2 }\end{array}$ & GNAS \\
\hline hsa-miR-374a & $\begin{array}{l}\text { CCL2, ADRB1, } \\
\text { WRN, WNT16 }\end{array}$ & $\begin{array}{l}\text { NTF3, PDPK1, PSMF1, SMAD6, } \\
\text { CEBPB, NMT1, FGFR2, TGFA, } \\
\text { DUSP6, EDAR, SOX4, WNT5A, } \\
\text { ABR, CCL2, ADRB1, WRN, } \\
\text { AKT1, BMP2, IL10, MSX1, } \\
\text { MAP2K4, MEF2D, }\end{array}$ & $\begin{array}{l}\text { WNT5A, ADAM10, CD47, NCK1, } \\
\text { PELI1, IL10, ING1, NUMB, CEBPB, } \\
\text { FGFR2, ASCL1, BMP2, CNOT8, } \\
\text { DLG3, EIF2S2, TFDP1, HES1, } \\
\text { MMP14, CCL2, WNT16, AKT1, } \\
\text { PITX2, TGFA, NR2F2, SOX4, }\end{array}$ & - \\
\hline hsa-miR-494 & $\begin{array}{l}\text { PTEN, BBC3, } \\
\text { CHEK2, CNR1, } \\
\text { SLC1A2, SIRT1 }\end{array}$ & $\begin{array}{l}\text { PTEN, BBC3, CHEK2, CNR1, } \\
\text { CLI3, IL12B, KLF11, ACVR1C, } \\
\text { GULP1, MTDH, PPARGC1A, } \\
\text { ROCK1, UACA, IGF1R }\end{array}$ & $\begin{array}{l}\text { PTEN, GLI3, IL12B, NFIB, } \\
\text { TACC2, IGF1R, DNAJA2, KLF11, } \\
\text { CKS1B, GPNMB, PBRM1, EVI5, } \\
\text { RAP1B, HHIP, SIRT1 LIF }\end{array}$ & - \\
\hline hsa-miR-181d & $\begin{array}{l}\text { ATM, PRKCD, } \\
\text { SERPINE1, TGFBR1, } \\
\text { ADRBK1, TIMP3 }\end{array}$ & $\begin{array}{l}\text { NOTCH2, PAWR, SRPK2, TNF, } \\
\text { BAG4, CBX4, CCNG1, DDIT4, } \\
\text { PRKCD, SERPINE1, TGFBR1, } \\
\text { ATM, RAD21, RNF34, BMP7, } \\
\text { GATA6, HEY2, IL1A, INSL3, } \\
\text { ITSN1, HSP90B1, TNFAIP1, } \\
\text { TRIM22, USP47 }\end{array}$ & $\begin{array}{l}\text { ATM, PRKCD, SERPINE1, TNF, } \\
\text { CBLB, CDON, ING5, CARD11, } \\
\text { GATA6, HEY2, INSL3, NOTCH2, } \\
\text { PAWR, PLAU, PRDM4, PROX1, } \\
\text { TGFBR1, BIRC6, IL1A, } \\
\text { RBBP7, TNS3 }\end{array}$ & - \\
\hline hsa-miR-532-5p & NUAK1 & $\begin{array}{l}\text { HSPA9, MED1, MBD4, IRS2, } \\
\text { LEP, CAPN3, CYC5, EYA2 }\end{array}$ & $\begin{array}{l}\text { LEP, NDP, PURA, SKAP2, MED1, } \\
\text { DDX11, IRS2, KRAS, FRS2 }\end{array}$ & - \\
\hline hsa-miR-622 & NPM1 & $\begin{array}{l}\text { NPM1, APPL1, E2F1, PPARD, } \\
\text { SATB1, BTK, SORT1, } \\
\text { RARG, DYRK2 }\end{array}$ & $\begin{array}{l}\text { PPARD, SATB1 FBXW7, RXRB, } \\
\text { TGIF1, RARG, NPM1, APPL1, } \\
\text { E2F1, SALL1, MBD2 }\end{array}$ & - \\
\hline hsa-miR-625 & - & GHRH, IGF1, ISL1 & $\begin{array}{l}\text { GHRH, ARIH2, HOXD13, } \\
\text { SPARC, IGF1, ISL1 }\end{array}$ & - \\
\hline hsa-miR-660 & - & TFAP2B, CDH13, HIPK1 & TFAP2B, CDH13, LIFR & TFAP2B \\
\hline
\end{tabular}

In keratinocytes, UV irradiation induces several molecular responses, such as pro-apoptotic signaling pathways; Ras-,
MAPK- and small GTPase-mediated signal transduction and immune responses (1). We re-sorted the target genes in 
Tables II and III into categories of molecular responses. As shown in Fig. 3, the miRNA target genes were highly involved in these types of responses. However, the level of involvement varied. The majority of target genes was functionally related to anti-apoptosis and cell proliferation regulation, whereas a limited number of target genes was related to MAPKK activity. Collectively, these findings suggest that TECAmediated UV protective properties may regulate molecular interplay between miRNAs and their target genes to influence apoptosis and cell proliferation.

\section{Discussion}

In the present study, we verified that $C$. asiatica protects keratinocytes against UVB-induced damage. WST-1 assays demonstrated that $C$. asiatica-induced effects were dosedependent. Low-doses of TECA induced proliferation, while high-doses of TECA induced cell death, indicating that $C$. asiatica has a dichotomous role in cell growth. The medical and pharmaceutical uses of $C$. asiatica are diverse. In human dermal fibroblasts, $C$. asiatica induces cell proliferation, collagen synthesis and anti-senescence $(24,27)$. Additionally, $C$. asiatica induced gastric ulcers and promoted epithelial cell proliferation in rats (28). However, $C$. asiatica has also shown anti-proliferative properties in solid cancers, such as melanoma, breast, liver and gastric cancers, as well as in keratinocytes (29-33). An anti-proliferative effect on keratinocytes was shown to be highest following treatment with $18.4 \pm 0.6 \mu \mathrm{g} / \mathrm{ml} C$. asiatica extract (33). We determined that $>10 \mu \mathrm{g} / \mathrm{ml}$ of TECA was anti-proliferative, however, the lower doses of TECA $(<5 \mu \mathrm{g} / \mathrm{ml})$ led to increased cell proliferation. This was recently confirmed by another group, which demonstrated that $C$. asiatica has concentration-dependent, reciprocal proliferative effects (34).

Hashim et al (25) reported that $C$. asiatica protects against UVB damage in human dermal fibroblasts. However, we demonstrated that the protective effects also extended to keratinocytes and investigated the impact on miRNA. Although protecting keratinocytes from UVB-induced damage has been widely researched using other molecules and gene-based molecular studies, a limited number of miRNA-based molecular studies have been undertaken. Recently, p63-miRNA feedback was identified as an important signaling pathway in keratinocyte senescence (35). Another study determined that the protective effect of baicalin on UVB-treated keratinocytes was mediated by miRNA expression modulation (36), indicating that miRNAs have important roles in keratinocyte proliferation. Therefore, our miRNA-based study regarding keratinocytes may provide important information regarding anti-UV therapeutics.

Zhou et al (19) recently characterized the miRNA profile in UVB-irradiated normal human keratinocytes. Interestingly, they displayed that miR-296-5p and miR-423-5p were downregulated and upregulated by UVB irradiation $\left(30 \mathrm{~mJ} / \mathrm{cm}^{2}\right.$, for 4 and $24 \mathrm{~h}$ ) respectively. We found that the expression levels of these miRNAs showed the opposite responses to C. asiatica treatment; miR-296-5p was significantly increased by 2.54 -fold and miR-423-5p was decreased by 1.65 -fold. These results indicate that these miRNAs may be novel
C. asiatica target miRNAs that protect keratinocytes against UVB-induced damage.

The miRNA target prediction and GO analysis revealed that a number of the target genes were involved in the apoptosis and cell proliferation pathways. MAPK-mediated signaling pathways are reportedly involved in UVB responses in keratinocytes (1). However, our data demonstrated that the target genes were less involved in MAPK-related signal transduction, suggesting that miRNA-based UVB protection pathways in keratinocytes may be mediated via anti-apoptosis and positive regulation of cell growth pathways, rather than through a MAPK pathway. In fact, although UV irradiation increases phosphorylation-mediated activation of MAPK proteins, including p38 MAPK, JNK and ERK1/2, the transcription and translation levels of these proteins are not altered by UVB irradiation (1), indicating that the MAPK genes do not interplay with $C$. asiatica-specific miRNAs involved in protecting keratinocytes from UVB-induced damage.

In conclusion, our findings suggest that $C$. asiaticamediated protective mechanisms are mediated by alterations in miRNA expression. Although further confirmative studies are required to verify miRNA alterations and their putative targets, these data provide meaningful information to further our understanding of the cellular responses in TECAmediated UVB protection in human keratinocytes.

\section{Acknowledgements}

We are grateful to the members of our research group for their support and advice regarding this study. This study was supported by the Ministry of Education, Science and Technology (grant 20110028646 to S.A.) of the Republic of Korea.

\section{References}

1. Muthusamy V and Piva TJ: The UV response of the skin: a review of the MAPK, NFkappaB and TNFalpha signal transduction pathways. Arch Dermatol Res 302: 5-17, 2010.

2. Clydesdale GJ, Dandie GW and Muller HK: Ultraviolet light induced injury: immunological and inflammatory effects. Immunol Cell Biol 79: 547-568, 2001.

3. Bender K, Gottlicher M, Whiteside S, Rahmsdorf HJ and Herrlich P: Sequential DNA damage-independent and -dependent activation of NF-kappaB by UV. EMBO J 17: 5170-5181, 1998.

4. Lewis DA and Spandau DF: UVB activation of NF-kappaB in normal human keratinocytes occurs via a unique mechanism. Arch Dermatol Res 299: 93-101, 2007.

5. Li N and Karin M: Ionizing radiation and short wavelength UV activate NF-kappaB through two distinct mechanisms. Proc Natl Acad Sci USA 95: 13012-13017, 1998

6. Yuan ZQ, Feldman RI, Sun M, et al: Inhibition of JNK by cellular stress- and tumor necrosis factor alpha-induced AKT2 through activation of the NF kappa B pathway in human epithelial Cells. J Biol Chem 277: 29973-29982, 2002.

7. Liu J, Yang D, Minemoto Y, Leitges M, Rosner MR and Lin A: NF-kappaB is required for UV-induced JNK activation via induction of PKCdelta. Mol Cell 21: 467-480, 2006.

8. Kim H, Kang JS and Lee WJ: The production IL-21 and VEGF in UVB-irradiated human keratinocyte cell line, HaCaT. Immune Netw 10: 75-80, 2010.

9. Chang DS, Seo SJ and Hong CK: The effect of amniotic membrane extract on the expression of iNOS mRNA and generation of $\mathrm{NO}$ in $\mathrm{HaCaT}$ cell by ultraviolet $\mathrm{B}$ irradiation. Photodermatol Photoimmunol Photomed 18: 280-286, 2002.

10. Ambros V and Lee RC: Identification of microRNAs and other tiny noncoding RNAs by cDNA cloning. Methods Mol Biol 265: $131-158,2004$. 
11. Cheng AM, Byrom MW, Shelton J and Ford LP: Antisense inhibition of human miRNAs and indications for an involvement of miRNA in cell growth and apoptosis. Nucleic Acids Res 33: 1290-1297, 2005.

12. Chen JF, Mandel EM, Thomson JM, et al: The role of microRNA-1 and microRNA-133 in skeletal muscle proliferation and differentiation. Nat Genet 38: 228-233, 2006.

13. Cho WJ, Shin JM, Kim JS, et al: miR-372 regulates cell cycle and apoptosis of ags human gastric cancer cell line through direct regulation of LATS2. Mol Cells 28: 521-527, 2009.

14. Holst LM, Kaczkowski B and Gniadecki R: Reproducible pattern of microRNA in normal human skin. Exp Dermatol 19: e201-e205, 2010.

15. Holst LM, Kaczkowski B, Glud M, Futoma-Kazmierczak E, Hansen LF and Gniadecki R: The microRNA molecular signature of atypic and common acquired melanocytic nevi: differential expression of miR-125b and let-7c. Exp Dermatol 20: 278-280, 2011.

16. Ichihara A, Jinnin M, Yamane $\mathrm{K}$, et al: microRNA-mediated keratinocyte hyperproliferation in Psoriasis vulgaris. $\mathrm{Br} \mathrm{J}$ Dermatol 165: 1003-1010, 2011.

17. Xu N, Brodin P, Wei T, et al: MiR-125b, a microRNA downregulated in psoriasis, modulates keratinocyte proliferation by targeting FGFR2. J Invest Dermatol 131: 1521-1529, 2011

18. Hildebrand J, Rutze M, Walz N, et al: A comprehensive analysis of microRNA expression during human keratinocyte differentiation in vitro and in vivo. J Invest Dermatol 131: 20-29, 2011.

19. Zhou BR, Xu Y, Permatasari F, et al: Characterization of the miRNA profile in UVB-irradiated normal human keratinocytes. Exp Dermatol 21: 317-319, 2012.

20. Brinkhaus B, Lindner M, Schuppan D and Hahn EG: Chemical, pharmacological and clinical profile of the East Asian medical plant Centella asiatica. Phytomedicine 7: 427-448, 2000.

21. Maquart FX, Chastang F, Simeon A, Birembaut P, Gillery P and Wegrowski Y: Triterpenes from Centella asiatica stimulate extracellular matrix accumulation in rat experimental wounds. Eur J Dermatol 9: 289-296, 1999.

22. Jayashree G, Kurup Muraleedhara G, Sudarslal S and Jacob VB: Anti-oxidant activity of Centella asiatica on lymphoma-bearing mice. Fitoterapia 74: 431-434, 2003.

23. Babu TD, Kuttan G and Padikkala J: Cytotoxic and anti-tumour properties of certain taxa of Umbelliferae with special reference to Centella asiatica (L.) Urban. J Ethnopharmacol 48: 53-57, 1995.
24. Kim YJ, Cha HJ, Nam KH, Yoon Y, Lee H and An S: Centella asiatica extracts modulate hydrogen peroxide-induced senescence in human dermal fibroblasts. Exp Dermatol 20: 998-1003, 2011.

25. Hashim P, Sidek H, Helan MH, Sabery A, Palanisamy UD and Ilham M: Triterpene composition and bioactivities of Centella asiatica. Molecules 16: 1310-1322, 2011.

26. Pillai RS, Bhattacharyya SN and Filipowicz W: Repression of protein synthesis by miRNAs: how many mechanisms? Trends Cell Biol 17: 118-126, 2007

27. Lu L, Ying K, Wei S, et al: Asiaticoside induction for cell-cycle progression, proliferation and collagen synthesis in human dermal fibroblasts. Int J Dermatol 43: 801-807, 2004

28. Guo JS, Cheng CL and Koo MW: Inhibitory effects of Centella asiatica water extract and asiaticoside on inducible nitric oxide synthase during gastric ulcer healing in rats. Planta Med 70: $1150-1154,2004$

29. Park BC, Bosire KO, Lee ES, Lee YS and Kim JA: Asiatic acid induces apoptosis in SK-MEL-2 human melanoma cells. Cancer Lett 218: 81-90, 2005.

30. Babykutty S, Padikkala J, Sathiadevan PP, et al: Apoptosis induction of Centella asiatica on human breast cancer cells. Afr J Tradit Complement Altern Med 6: 9-16, 2008.

31. Lin LT, Liu LT, Chiang LC and Lin CC: In vitro anti-hepatoma activity of fifteen natural medicines from Canada. Phytother Res 16: 440-444, 2002

32. Yoshida M, Fuchigami M, Nagao T, et al: Antiproliferative constituents from Umbelliferae plants VII. Active triterpenes and rosmarinic acid from Centella asiatica. Biol Pharm Bull 28 $173-175,2005$.

33. Sampson JH, Raman A, Karlsen G, Navsaria H and Leigh IM: In vitro keratinocyte antiproliferant effect of Centella asiatica extract and triterpenoid saponins. Phytomedicine 8: 230-235, 2001.

34. Ruszymah BH, Chowdhury SR, Manan NA, Fong OS, Adenan MI and Saim AB: Aqueous extract of Centella asiatica promotes corneal epithelium wound healing in vitro. J Ethnopharmacol 140: 333-338, 2012.

35. Rivetti di Val Cervo P, Lena AM, Nicoloso M, et al: p63-microRNA feedback in keratinocyte senescence. Proc Natl Acad Sci USA 109: 1133-1138, 2012

36. Xu Y, Zhou B, Wu Di, Yin Z and Luo D: Baicalin modulates microRNA expression in UVB irradiated mouse skin. J Biomed Res 26: 125-134, 2012. 